\title{
Response differentiation: A psychophysical method for response produced stimuli'
}

\author{
DOUGLAS P. FERRARO ${ }^{2}$ AND DAVID M. GRILLY \\ UNIVERSITY OF NEW MEXICO
}

Twenty-four rats were reinforced for releasing a lever between $t$ and $t+.20 \mathrm{sec}$ after pressing it. Response duration differentiation performance decreased monotonically as $t$ was increased in length from $.10 \mathrm{sec}$ to $1.60 \mathrm{sec}$. A response analogue of Weber's ratio was constant throughout the middle range of duration bandwidth requirements and increased at the extremely short bandwidth-a response-differentiation finding analogous to Weber's law of stimulus discrimination. This finding was interpreted as support for the conceptualization that response differentiation represents a special instance of response discrimination or discrimination based on response-produced stimuli.

The usual discrimination paradigm involves reinforcing responses only in the presence of specified exteroceptive stimuli. Recent experiments (e.g., Pliskoff \& Goldiamond, 1966; Rilling \& McDiarmid, 1965) have demonstrated the acquisition of discriminations in situations where S's behavior replaces the exteroceptive stimulus in the stimulus-control paradigm, i.e., where $S$ must base its discrimination on specified prior behavior rather than on E-controlled exteroceptive stimuli. Such response discriminations may be considered as stimulus discriminations if it is assumed that response discriminations are based on some unspecified response-produced stimuli. Thus, the discrimination paradigm may be expanded to include reinforcing responses only in the presence of stimuli produced by prior behavior.

Regardless of whether stimuli are $E$ or response produced, the stimulus-discrimination paradigm is operationally distinguishable from the response-differentiation paradigm. The latter paradigm involves making reinforcement contingent on the occurrence of specified qualitative or quantitative (e.g., force, duration) properties of the response. Notterman and Mintz (1965) have hypothesized that responses that differ quantitatively produce quantitatively different interoceptive stimuli, the discrimination of which is necessary to perform differentially quantitative response variants. In this context, the response differentiation paradigm reduces to reinforcing responses only in the presence of those interoceptive stimuli correlated with specific quantitative response values. Accordingly, response differentiation may be conceptualized as a special instance of response discrimination or, more generally, of discrimination.

Viewed as discrimination, response differentiation represents a psychophysical method for examining response-produced stimuli that is analogous to the Method of Adjustment for exteroceptive stimuli. S's task is to adjust its response quantitatively until the stimuli produced by that response are discriminated as equal to the standard stimuli, that is, to those stimuli produced by the criterion response for reinforcement. The standard deviation of the distribution of obtained quantitative response variants serves as a discrimination measure of response-produced stimuli, and the ratio, standard-deviation/criterionresponse value, is the response analogue of Weber's ratio.

If the differentiation is subsumed by discrimination, then phenomena obtained with discrimination procedures should be demonstrable using analogous differentiation procedures (Ferraro, Grilly, \& Tang, 1968). Specifically, it should be possible to demonstrate Weber's law in the context of response differentiation. Therefore, the present experiment was designed to test the prediction that a response must be increased by a constant fraction of its quantitative value to be differentiated equally. The experiment was performed along a response duration continuum and employed a duration bandwidth differentiation procedure (Taber, Homme, \& Csanyi, 1961), in which only response durations longer than length $t$, but shorter than length $t+n$, were reinforced at several values of $t$.

\section{Subjects}

\section{METHOD}

Twenty-four naive male Long-Evans rats from the Simonsen breeding colony, 200 days old at the beginning of experimentation, were allowed free access to food in their home cages and were maintained at $80 \%$ of ad lib weight by limiting water intake.

\section{Apparatus}

The experimental chambers were two identical Scientific Prototype rat boxes equipped with a single response lever (1.9 cm long, $.6 \mathrm{~cm}$ diam) and a Lehigh Valley liquid dipper programmed to deliver $.02 \mathrm{cc}$ of water reinforcement for $3 \mathrm{sec}$. A 15-g downward force, applied to the lever for $.4 \mathrm{~cm}$, closed the response microswitch. Response duration was defined as the length of time the microswitch remained closed. Duration distributions were obtained for each $S$ during every experimental session by distributing response durations into $18.10-\mathrm{sec}$ - or $.20-\mathrm{sec}$-wide categories with the use of transistorized digital logic and electromagnetic counters. All response durations greater than the 18th category $(1.8 \mathrm{sec}$ or $3.6 \mathrm{sec})$ were cumulated in a separate counter. A minimum duration of $50 \mu \mathrm{sec}$ was required to activate the programming apparatus. All reinforcements were delivered after the response microswitch was opened.

\section{Procedure}

All Ss were trained to make the bar-press release response and allowed 50 reinforcements on a schedule in which every response was reinforced regardless of duration. In the subsequent session, each $S$ was given 75 additional reinforcements on the regular reinforcement schedule. After this preliminary training, four Ss were randomly assigned to each of six experimental groups and a duration bandwidth restriction was superimposed on the regular reinforcement base-line schedule; only those bar releases that followed bar presses by at least $t$ sec but less than $t+.20 \mathrm{sec}$ were reinforced. Each group was exposed to a single value of $t$ $(.10, .30, .50, .70,1.20$, or $1.60 \mathrm{sec})$ for 14 consecutive daily sessions. Sessions 1 and 2 were terminated after 50 reinforcements were delivered and Sessions 3-14 after 100 reinforcements, so that each $S$ received a total of 1,300 bandwidth reinforcements.

\section{RESULTS}

The percentages of total bar releases falling between $t$ and $t+.20 \mathrm{sec}$ were averaged across the first (Sessions 1.5) and last (Sessions 11-14) 400 reinforcements and are separately presented for each 


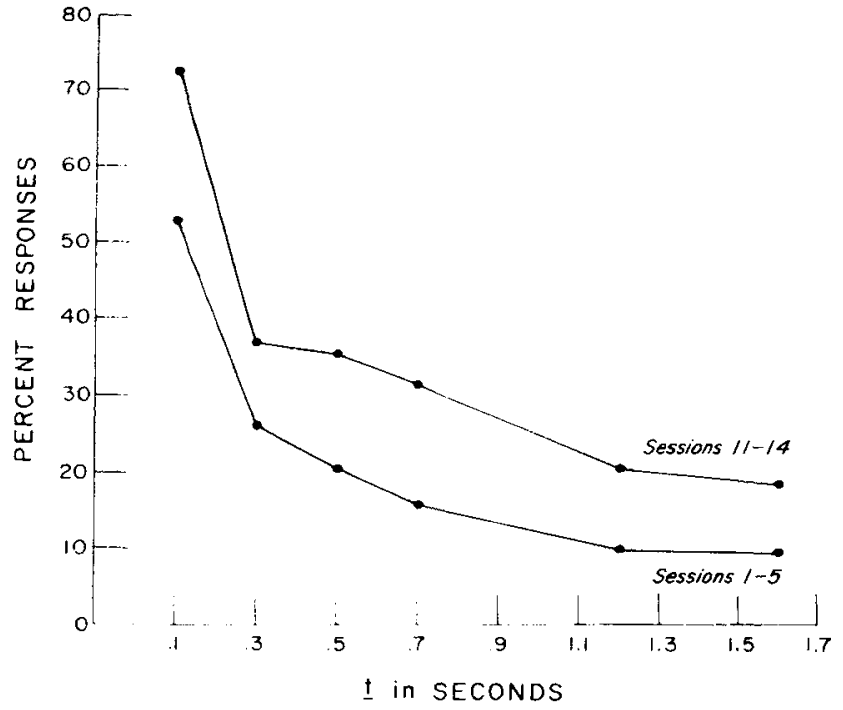

duration bandwidth requirement in Fig. 1. The percentage of within-bandwidth responses at each $t$ value increased with continued exposure to the duration bandwidth requirement. However, differentiation performance during both early and late training was a decreasing monotonic function of the bandwidth requirement. An analysis of variance performed on the percentage of bandwidth responses for each $t$ value over Sessions 11-14 showed that the obtained bandwidth differences were significant $(\mathrm{F}=16.13, \quad \mathrm{df}=5 / 18, \quad \mathrm{p}<.001) . \quad$ In addition, both significant linear ( $F=63.63$, $\mathrm{df}=1 / 18, \quad \mathrm{p}<.001)$ and quadratic $(F=9.68, \mathrm{df}=1 / 18, \mathrm{p}<.01)$ components were obtained and, thus, support the curvilinear function relating differentiation performance to bandwidth requirement depicted in Fig. 1.

That the improvement in differentiation performance observed across sessions at each $t$ value was due to changes in both the central tendency and variability of the obtained response duration distributions is shown in Table 1. Except for $t=.10 \mathrm{sec}$, which was overestimated, the median response duration obtained in Sessions 1-5 was shorter than the required $t$ value. Furthermore, the amount by which the standard (reinforced) response was underestimated was directly related to the
Fig. 1. Percentage of total response durations longer than $t$ sec but less than $t+.20 \mathrm{sec}$ plotted separately for Sessions 1-5 and Sessions 11-14 as a function of $t$.

to Weber's ratio, serves as an index of the difference threshold for response-produced stimuli under the present procedure. During initial exposure to the differentiation requirements, $\Delta D / D$ was smallest at the intermediate bandwidth requirement and increased at the extremely short and long bandwidths. However, with continued training, $\Delta \mathrm{D} / \mathrm{D}$ decreased at all bandwidth requirements and appeared to have approached constancy $(.375 \mathrm{sec})$ throughout the range of bandwidth requirements greater than $.10-.30 \mathrm{sec}$. An analysis of variance across Sessions 11-14 showed that the overall differences in the $\Delta D / D$ ratios obtained at each $t$ value were significant $(F=4.186, d f=5 / 18$, $p<.025)$. However, the only pairwise comparisons to yield significance at the .05 level under Duncan's multiple range test were those between $t=.10 \mathrm{sec}$ and $t$ $>.10 \mathrm{sec}$.

\section{DISCUSSION}

The finding that differentiation performance is a decreasing function of the absolute magnitude of the standard response ( $t)$ is in accord with prior data obtained with human Ss under somewhat comparable differentiation procedures (e.g., Bahrick \& Noble, 1961; Mintz \& Notterman, 1965; Taber et al, 1961). Several analogies between the present data and data typically obtained with psychophysical discrimination procedures are apparent. For example, the finding that median duration was greater than $t$ at the low $t$ value and less than $t$ at larger $t$ values is analogous to the range effect and constant time error typically found in psychophysical estimations (Woodworth \& Schlosberg, 1954). Similarly, the findings that duration variability was proportional to the value of $t$ and that $\Delta D / D$ was constant across a wide range of duration bandwidth requirements are analogous to the familiar Weber's law of discrimination. As predicted, these latter findings suggest that equal-duration differentiations may be obtained at several $t$ values by increasing the width of the duration band requirement by a constant fraction of $t$.

As suggested previously (Ferraro et al, 1968), similarities between data obtained with analogous differentiation and discrimination procedures lend indirect support to the notion that response differentiation represents the special case of stimulus discrimination in which the stimuli that, in part, define the 


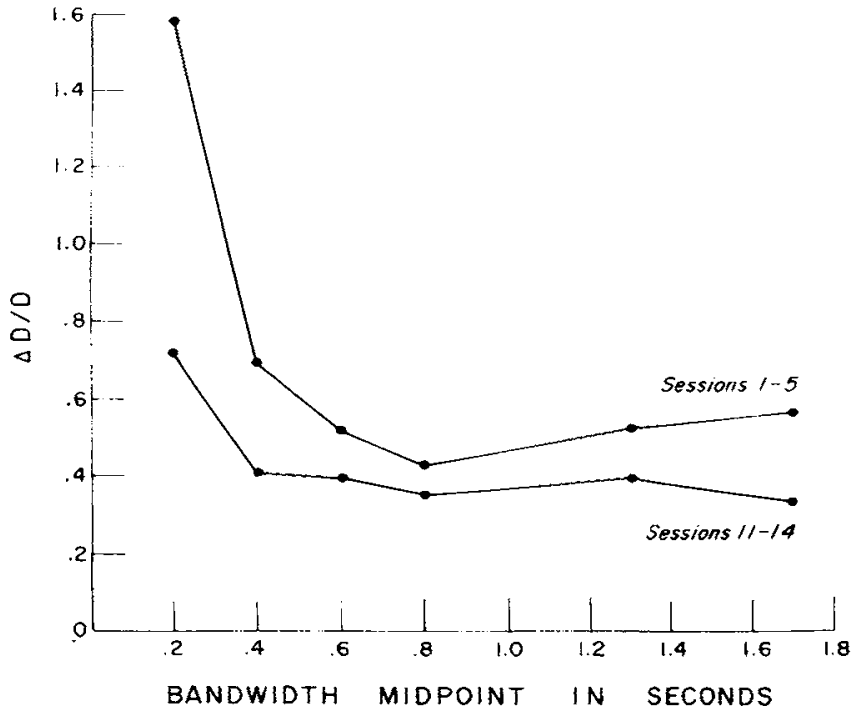

reinforcement contingency arise from the response itself. Grice (1965), among others, has argued that conceptualizations that place the weight of explanation on response-produced stimuli do not necessarily lead to specification of either the identity or properties of these stimuli. In this context, it is suggested that the present data indicate that response-produced stimuli, whatever their identity, have properties common to other (exteroceptive) stimuli.

\section{REFERENCES} 793-796.
Fig. 2. Average ratio of duration dispersion to the midpoint of the required duration bandwidth $(\Delta D / D)$ plotted separately for Sessions 1-5 and Sessions 11.14 as a function of the bandwidth midpoint.

NOTTERMAN, J. M., \& MINTZ, D. E. Dynamics of response. New York: Wiley, 1965.

PLISKOFF, S. S., \& GOLDIAMOND, I. Some discriminative properties of fixed ratio performance in the pigeon. Journal of the Experimental Analysis of Behavior, 1966, 9, 1-9.

RILLING, M., \& McDIARMID, C. Signal detection in fixed ratio schedules. Science, $1965,148,526-527$.

TABER, J. I., HOMME, L. E., \& CSANYI, A. P. The differentiation of human time estimations. Journal of the Experimental Analysis of Behavior, 1961, 4, 299-304.

WOODWORTH, R. S., \& SCHLOSBERG, H. Experimental psychology. New York: Holt, 1954.

BAHRICK, H. P., \& NOBLE, M. On stimulus and response discriminability. Joumal of Experimental Psychology, 1961, 61, 449-454. FERRARO, D. P., GRILLY, D. M., \& TANG, R. S. W. Transfer of a differentiation along a response continuum. Journal of Comparative \& Physiological Psychology, 1968, 66,

GRICE, G. R. Do responses evoke responses? American Psychologist, 1965, 20, 282-294.

MINTZ, D. E., \& NOTTERMAN, J. M. Force differentiation in human subjects. Psychonomic Science, 1965, 2, 289-290.

\section{NOTES}

1. This research was supported by Grant GB-7674 from the National Science Foundation to D. P. Ferraxo. The authors thank A. H. Wolach Grice for his comments on an earlier version of this paper.

2. Address: Department of Psychology, The University of New Mexico, Albuquerque, New Mexico 87106.

(Accepted for publication May 14, 1969.) for his help in collecting the data and Dr. G. R. 